\title{
Recreating gut-liver axis during NAFLD onset by using a Caco-2/HepG2 co-culture system
}

\author{
Marica Meroni ${ }^{1}$, Erika Paolini ${ }^{1,2}$, Miriam Longo ${ }^{1,3}$, Roberto Piciotti ${ }^{1,4}$, Giada Tria ${ }^{1}$, Silvia Fargion ${ }^{1}$, Anna \\ Ludovica Fracanzani $^{1,4}$, Paola Dongiovanni ${ }^{1}$ \\ 'General Medicine and Metabolic Diseases, Fondazione IRCCS Ca' Granda Ospedale Maggiore Policlinico, Milan 20122, Italy. \\ ${ }^{2}$ Department of Pharmacological and Biomolecular Sciences, Università degli Studi di Milano, Milano 20122, Italy. \\ ${ }^{3}$ Department of Clinical Sciences and Community Health, Università degli Studi di Milano, Milano 20122, Italy. \\ ${ }^{4}$ Department of Pathophysiology and Transplantation, Università degli Studi di Milano, Milano 20122, Italy.
}

Correspondence to: Dr. Paola Dongiovanni, General Medicine and Metabolic Diseases, Fondazione IRCCS Ca' Granda Ospedale Maggiore Policlinico, Milan 20122, Italy. E-mail: paola.dongiovanni@policlinico.mi.it

How to cite this article: Meroni M, Paolini E, Longo M, Piciotti R, Tria G, Fargion S, Fracanzani AL, Dongiovanni P. Recreating gutliver axis during NAFLD onset by using a Caco-2/HepG2 co-culture system. Metab Target Organ Damage 2022;2:4. https://dx.doi.org/10.20517/mtod.2021.19

Received: 23 Dec 2021 First Decision: 25 Jan 2022 Revised: 27 Jan 2022 Accepted: 10 Feb 2022 Published: 22 Feb 2022

Academic Editors: Annalisa Berzigotti, Claudio Tiribelli Copy Editor: Xi-Jun Chen Production Editor: Xi-Jun Chen

\begin{abstract}
Nonalcoholic fatty liver disease (NAFLD) onset and its progression towards nonalcoholic steatohepatitis (NASH) features increased intestinal permeability and leaky gut, thereby favoring the escape of endotoxin [lipopolysaccharides (LPS)] from the gut to the liver. The aim of this study was to resemble the crosstalk between intestine and liver during NAFLD by using an in vitro model of co-culture system. Enterocytes (Caco-2) were seeded on Transwell filters (pore size: $0.4 \mu \mathrm{m}$ ) and cultured for 21 days to constitute a confluent monolayer, and then they were co-cultivated with hepatocytes (HepG2) for an additional $24 \mathrm{~h}$. Caco-2 on the apical chamber were exposed to LPS and/or a mixture of palmitic and oleic acid (PAOA) for $24 \mathrm{~h}$. FITC-4000 dextrans (FD4) permeability across Caco-2 monolayer was increased by the treatment of Caco-2 cells with PAOA and LPS, consistently with tight junction-associated proteins reduction. Caco-2 exposure to PAOA/LPS promoted ApoB, triglyceride (TG), and free fatty acid secretion in basolateral media. In turn, HepG2 co-cultured with Caco-2 exposed to LPS, PAOA, or both accumulated lipid droplets and increased intracellular TG content. Likewise, Caco-2 released pro-inflammatory cytokines in basolateral media. These events triggered endoplasmic reticulum (ER) and oxidative stress, enhancing reactive oxygen species (ROS), $\mathrm{H}_{2} \mathrm{O}_{2}$, aldehyde derivate production, and ROS-induced DNA damage in HepG2 cells. Hence, Caco-2/HepG2 co-culture system may faithfully reproduce the breach in the intestinal barrier integrity that occurs in NAFLD, thus resulting in the increased inflammatory response and ER and
\end{abstract}


oxidative and stress, which promote the switch towards NASH.

Keywords: NAFLD, NASH, gut-liver axis, intestinal permeability, leaky gut, co-culture system

\section{INTRODUCTION}

Nonalcoholic fatty liver disease (NAFLD), or as it has been more recently re-named "metabolic dysfunction-associated fatty liver disease (MAFLD)" ${ }^{[1]}$, is the most frequent chronic hepatic disorder worldwide, affecting $20 \%-40 \%$ of the adult population in Western countries ${ }^{[2,3]}$. Hence, due to its ever rising prevalence, it constitutes a huge socioeconomic burden ${ }^{[4]}$. NAFLD is diagnosed when hepatic fat accumulation exceeds $5 \%$ of liver weight in absence of alcohol misuse, and it entails a wide phenotypic variety of hepatic aberrancies, ranging from simple and uncomplicated steatosis to nonalcoholic steatohepatitis (NASH), in which steatosis is intertwined with inflammation, oxidative stress, and hepatocyte degeneration. This condition may be aggravated by fibrosis, and, in some cases, it may worsen into cirrhosis and hepatocellular carcinoma $(\mathrm{HCC})^{[5,6]}$. The convoluted natural course of the disease relies on multiple drivers, encompassing exacerbated immune system activation, endoplasmic reticulum (ER) and oxidative stress, organelle impairment, and DNA derangements ${ }^{[7]}$. However, the fine-tuned mechanisms which regulate the switching from fatty liver to NASH are still under study.

In this context, mounting evidence points to the gut-liver axis as one of the primary contributors to NAFLD development and its precipitation to NASH up to more severe forms, as a consequence of the close anatomical and functional relationship between the gastrointestinal tract and the liver ${ }^{[8,9]}$. Indeed, the hepatic tissue is exposed to a perpetual flow of potentially harmful gut microbial byproducts and ingested nutrients via the portal vein, and, consequently, the liver modulates microbiota taxonomic composition through bile acid secretion into the duodenum ${ }^{[8]}$. The bowel wall displays immunological and selective barrier properties, regulating bidirectional fluxes between the gut and the liver, due to the presence of adherent and tight junctions [constituted by zonula occludens 1 (ZO-1) and claudins] and desmosomes, which hold together the juxtaposed epithelial cells and protects the host from invasive bacteria, thus avoiding pathogens overgrowth and their systemic translocation ${ }^{[8,10]}$. Notwithstanding, defects in intestinal barrier integrity (a phenomenon known as leaky gut) along with imbalances in gut microbiota proportion (referred to as dysbiosis) frequently affect patients with severe NAFLD ${ }^{[1,12]}$. As a result, enhanced gut permeability has been associated with the escape of pathogenic microorganisms and endotoxemia, thereby fostering intestinal mucosal inflammation and a chronic low-grade immune system activation, as reported in several studies ${ }^{[13-16]}$. Therefore, disturbances in the gut-liver axis homeostasis may describe the evolution of steatosis to NASH and $\mathrm{HCC}^{[17-21]}$. Lipopolysaccharides (LPS) per se, including several pathogen-associated molecular patterns, activate toll-like receptor (TLR) cascade in hepatocytes, Kupffer cells, and hepatic stellate cells, elevating serum tumor necrosis factor alpha (TNF- $\alpha$ ) and interleukin 6 (IL6) and precipitating systemic inflammation and fibrosis ${ }^{[22,23]}$. Otherwise, exaggerated amounts of free fatty acids (FFAs), whose levels are typically increased in NAFLD due to enhanced adipose tissue lipolysis, may independently modulate inflammatory pathways ${ }^{[24,25]}$. In turn, severe inflammation in NASH patients may contribute to favor intestinal high-permeability and dysbiosis ${ }^{[26]}$. In addition, similar to pro-inflammatory mediators, microRNAs (miRNAs) and dysregulated bile acid secretion may also influence gut barrier integrity by targeting ZO-1, claudins, and occludins ${ }^{[27]}$. However, intensive efforts are essential to completely understand the true causality between intestinal impairments and hepatic morbidities as well as to pinpoint the mechanisms through which intestinal alterations may affect liver pathology. 
Thus, to model the interaction which occurs between the gut and the liver in the context of NAFLD, a representative in vitro co-culture system is necessary. Unfortunately, these studies may often require sophisticated and expensive intestine-liver-on-chip platforms ${ }^{[28]}$, partially limiting their wide applications. Here, our aim was to exploit a reproducible protocol to possibly integrate different signals that participate to the onset of NAFLD and its progression towards NASH. Since increased intestinal permeability along with systemic LPS translocation represents the hallmarks of progressive NAFLD, featuring excessive FFA efflux to the liver, we aimed to recreate the gut-liver axis interactions by exploiting an in vitro model of a coculture system of enterocytes (Caco-2) and hepatocytes (HepG2). Caco-2 on the apical chamber were exposed to LPS and/or a mixture of palmitic and oleic acid (PAOA) to recapitulate what occurs in NAFLD due to the presence of dysbiosis and higher fat intake. Then, we measured intestinal permeability and lipid and cytokine secretion in basolateral media. As concern the hepatic response, it was evaluated in terms of fat overload, inflammation, and ER and oxidative stress.

\section{MATERIALS AND METHODS}

\section{Reagents}

Dulbecco's modified Eagle's medium (DMEM), phosphate-buffered saline (PBS), fetal bovine serum (FBS), L-glutamine, penicillin/streptomycin, Trypsin/EDTA, Hank's balanced salt solution, and fast SYBR green master mix were obtained from ThermoFisher Scientific (Waltham, USA). Cell culture flasks, plates, and polyester membrane inserts ( $12 \mathrm{~mm}$ diameter, $0.4 \mu \mathrm{m}$ pore diameter) were obtained from Corning (Cambridge, MA, USA). Free fatty acids (FFAs) Quantification Kit and Triglyceride Quantification Kit were purchased from BioVision (Milpitas, United States). Cholesterol Colorimetric Assay Kit - HDL and LDL/VLDL, DCF ROS/RNS Colorimetric Assay Kit, Hydrogen Peroxide Assay Kit, Lipid Peroxidation (MDA) Assay Kit, and DNA Damage Assay Kit were purchased from Abcam (Cambridge, UK). AntipTLR4, TLR4, ZO-1, and Claudin antibodies were acquired from Cell Signaling Technologies (Boston, USA). Anti $\beta$-Actin was purchased from Abcam (Cambridge, UK).

\section{Cell culture and co-culture system}

Caco-2 cells, a model of the human colon epithelial cancer cell line, were obtained from ATCC (HTB-37, LGC Standards, Milan, Italy) and were cultured at 50\% confluency and maintained at $37^{\circ} \mathrm{C}$ under $5 \% \mathrm{CO}_{2}$ atmosphere in DMEM containing $1 \%$ L-glutamine, $100 \mathrm{U} / \mathrm{L}$ penicillin, and $100 \mu \mathrm{g} / \mathrm{L}$ streptomycin, supplemented with $10 \%$ FBS. For Caco-2 differentiation, cells were seeded on polycarbonate filter Transwells with $12 \mathrm{~mm}$ of diameter and $0.4 \mu \mathrm{m}$ semipermeable pore diameter (Transwell, Corning Inc., Lowell, MA, USA) at a $3.5 \times 10^{5} \mathrm{cells} / \mathrm{cm}^{2}$ density in complete medium supplemented with $10 \% \mathrm{FBS}$ in both apical and basolateral compartments for two days to allow the formation of a confluent cell monolayer. Then, from the third day after seeding, fresh complete medium was added in both compartments and cells were cultured for 21 days with regular medium changes three times per week. During these 21 days, Caco-2 spontaneously differentiated into small intestinal phenotype, acquiring microvilli at the apical pore and formed tight intercellular junctions at the basolateral surface, thus mimicking the human intestinal absorption $^{[29]}$.

The HepG2 cell line was provided by from ATCC (HB-8065, LGC Standards, Milan, Italy), and it was cultured in DMEM supplemented with $10 \% \mathrm{FBS}, 100 \mathrm{U} / \mathrm{mL}$ penicillin, $100 \mu \mathrm{g} / \mathrm{mL}$ streptomycin, and $1 \% \mathrm{~L}$ glutamine and incubated at $37{ }^{\circ} \mathrm{C}$ under $5 \% \mathrm{CO}_{2}$ atmosphere. Caco-2 and HepG2 cells were used for no more than 20 passages after thawing, as the increase in the number of passages may affect cell phenotype and alter experimental results. 


\section{Cell monolayer integrity and differentiation evaluation}

To determine whether Caco-2 cell layers were fully confluent, Transwells were placed in new multi-well plates and then phenol red leakage, through the intercellular spaces, from the apical to the basolateral compartment was examined ${ }^{[30]}$. DMEM with phenol red was added to the apical compartment, while prewarmed PBS $1 \times$ without phenol red was added to the basolateral compartment for $2 \mathrm{~h}$ at $37^{\circ} \mathrm{C}$ under $5 \%$ $\mathrm{CO}_{2}$ atmosphere. The optical density (OD) of the basolateral solution was measured at $450 \mathrm{~nm}$ using a spectrophotometer (Beckman, Pasadena, CA, USA) and only confluent Caco-2 polarized monolayers (characterized by a measure of OD at $450 \mathrm{~nm}$ of the basolateral medium less than 0.02 ) were used for coculture experiments ${ }^{[31]}$.

\section{In vitro treatments}

HepG2 cells were seeded at the concentration of $3.5 \times 10^{5}$ in multi-well plates and cultured for $48 \mathrm{~h}$. Then, confluent monolayers of intestinal Caco-2 cells on Transwells were transferred on top of multi-well culture plates containing 90\% confluent HepG2 cells, at the bottom. Caco-2 and HepG2 cells were incubated together in a co-culture system to recreate the bidirectional interplay between gut and liver. After an overnight quiescence in DMEM, 0.5\% FBS, $100 \mathrm{ng} / \mathrm{mL}$ LPS (Sigma-Aldrich, Amsterdam, The Netherlands), and/or a mixture of palmitic and oleic acids at the final concentration of $0.25 \mathrm{mM}$ (ratio 1:2, Sigma-Aldrich) were added in the quiescent medium of the apical chamber, whereas fresh quiescent medium was added to the basolateral compartment. After $24 \mathrm{~h}$ of incubation, co-cultured cells and apical and basolateral media were collected for further analyses. The optimal LPS and FFAs concentrations were determined by previous experiments ${ }^{[32]}$. A schematic representation of the Caco-2/HepG2 co-culture system is illustrated in Figure 1.

\section{Epithelial permeability assay}

To test the integrity of Caco-2 confluent monolayer, which mimics the intestinal barrier ${ }^{[33]}$, in response to FFAs and/or LPS stimulation, we measured the transepithelial flux of $4 \mathrm{kDa}$ fluorescein isothiocyanate (FITC)-labeled dextrans (FD4, Sigma Aldrich) as previously described ${ }^{[3,35]}$. Briefly, intestinal monolayers were equilibrated with pre-warmed PBS $1 \times$ at $37^{\circ} \mathrm{C}$, and then the fluorescent FD4 $(1 \mathrm{mg} / \mathrm{mL}$ in PBS $1 \times)$ was added to the apical chamber. Then, $100 \mu \mathrm{L}$ of sample were removed from the basolateral compartment at 1 $\mathrm{h}$ intervals for $4 \mathrm{~h}$, and the volume removed was replaced with warmed PBS $1 \times$. Epithelial permeability was compared between the different experimental conditions in quadruplicate, at the end of the $4 \mathrm{~h}$. Fluorescence intensity (excitation $492 \mathrm{~nm} /$ emission $520 \mathrm{~nm}$ ) was measured on a Promega GloMax plate reader. Concentrations of FD4 passed through the gut barrier were determined by linear regression using known dilutions of FITC-dextrans in PBS $1 \times$.

\section{Lipid secretion}

Basolateral media of co-cultured cells were collected and concentrated using the Vivaspin 20/10 KDa polyethersulfone twin membrane (Sartorius Stedim Biotech, France), with the purpose to quantify apolipoprotein $\mathrm{B}(\mathrm{ApoB})$ release through enzyme linked immunosorbent assay (ELISA), which detects both ApoB main isoforms, the intestinal ApoB-48 and the hepatic ApoB-100, according to the manufacturer's instructions (ThermoFisher Scientific). The lower limit of ApoB detection was $0.21 \mathrm{ng} / \mathrm{mL}$.

The same methodological procedure was applied to determine TG-lipoprotein, TG, and FFA concentrations in basolateral media, by using sensitive enzyme-based methods (Abcam, Cambridge, UK).

All quantifications were normalized for the total protein content. 


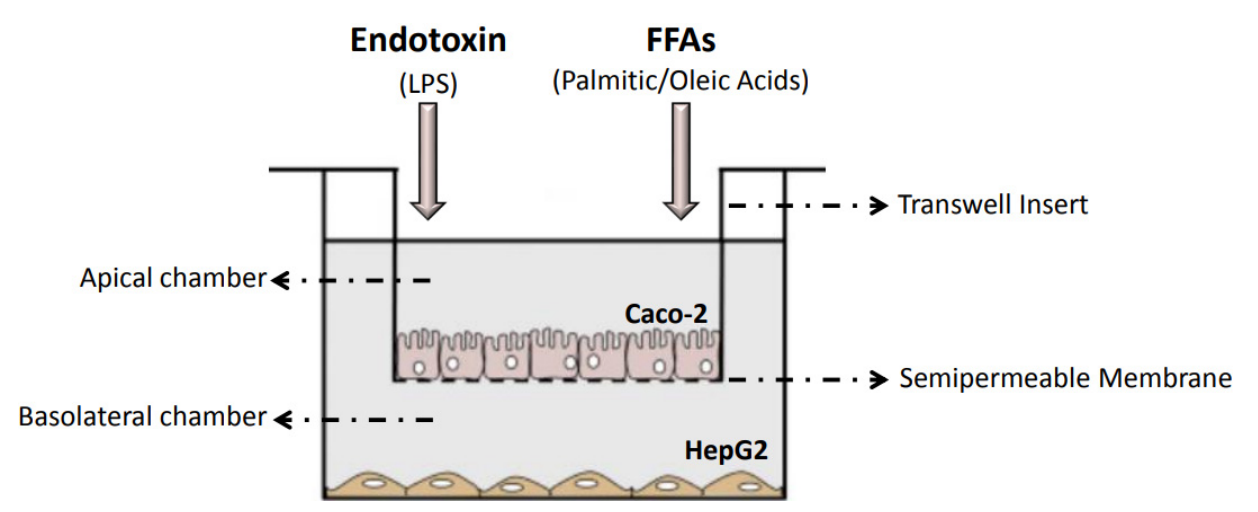

Figure 1. Schematic overview of the Caco-2/HepG2 co-culture system. Caco-2 cells were grown and differentiated on polycarbonate filter membranes ( $0.4 \mu \mathrm{m}$ semipermeable pore diameter) for 21 days to recreate intestinal barrier. Then, Transwells were transferred on the top of confluent HepG2 cells seeded in multi-well plates and treated with endotoxin (LPS) and/or palmitic and oleic acids (FFAs) to mimic gut-liver interplay during NAFLD onset. NAFLD: Nonalcoholic fatty liver disease.

\section{Cytokine secretion}

Basolateral concentrated media were also exploited to quantify cytokine secretion through ELISA assays to detect TNF- $\alpha$, IL6, and IL1 $\beta$ (R\&D Systems, Minneapolis, USA). The lower limits of detection were 15.6, 3.9, and $9.4 \mathrm{pg} / \mathrm{mL}$, respectively. Data were normalized to the total protein content.

\section{Quantification of DNA damage}

Total DNA was extracted from cell lysates of Caco2 and HepG2 by using DNeasy Blood \& Tissue Kits (Qiagen, Hilden, Germany). Then, oxidative DNA damage induced by free radicals and other reactive species was measured as the formation of a-basic sites in genomic DNA, exploiting an aldehyde reactive probe that interacts with an aldehyde group on the open ring form of apurinic/apyrimidinic (AP) sites. The latter are common DNA lesions developed in the presence of enhanced oxidative stress. AP sites are tagged with biotin residues that can later be quantified using a streptavidin-enzyme conjugate, detected at OD450 $\mathrm{nm}$ (sensitivity range of 4-40 AP sites per $1 \times 10^{5} \mathrm{bp}$ ). Absorbances were normalized to the total amount of DNA isolated for each condition ${ }^{[36,37]}$.

\section{Gene expression analysis}

RNA was extracted from cell cultures using Trizol reagent (ThermoFisher Scientific, Waltham, USA). Then, $1 \mu \mathrm{g}$ of total RNA was retro-transcribed with VILO random hexamers synthesis system (ThermoFisher Scientific), and real-time quantitative reverse transcription PCR (qRT-PCR) was performed with a QuantStudio 3 Real-Time PCR thermocycler (ThermoFisher Scientific) using SYBR Green chemistry (Fast SYBR Green Master Mix; ThermoFisher Scientific) for all genes following the manufacturer's instructions. All reactions were delivered in triplicate. Data were normalized for $\beta$-actin gene expression, and the results were expressed as arbitrary units or fold increase as indicated in violin plots. All primers are listed in Table 1.

\section{Western blot analysis}

Total protein lysates were extracted from co-cultures using RIPA buffer containing phosphatase and protease inhibitors (PhosSTOP and cOmplete ${ }^{m}$ Protease Inhibitor Cocktails, Roche-Sigma Aldrich). Samples were pooled prior to electrophoretic separation and all reactions were performed in duplicate. Then, equal amounts of proteins $(60 \mu \mathrm{g})$ were separated by SDS-PAGE, transferred electrophoretically to nitrocellulose membrane (BioRad, Hercules, CA), and incubated with specific antibodies overnight. At least three independent lots of freshly extracted proteins were used for experiments. The antibodies and concentrations used are listed in Table 2. 
Table 1. Sequence of primers used in quantitative real-time PCR experiments

\begin{tabular}{|c|c|c|}
\hline & Forward $5^{\prime} \rightarrow 3^{\prime}$ & Reverse $5^{\prime} \rightarrow 3^{\prime}$ \\
\hline ATF4 & AAACCTCATGGGTTCTCCAG & GGCATGGTTTCCAGGTCACT \\
\hline ATF6 & AATTCTCAGCTGATGGCTGT & TGGAGGATCCTGGTGTCCAT \\
\hline FASn & GCTGGGTGGAGTCTCTGAAG & TGCAACACCTTCTGCAGTTC \\
\hline GRP78 & CTTGCCGTTCAAGGTGGTTG & CTGCCGTAGGCTCGTTGAT \\
\hline MnSOD2 & CAAATTGCTGCTTGTCCAAA & TCTTGCTGGGATCATTAGGG \\
\hline MTTP & GCTTCCGTTAAAGGTCACACA & CAGCTGTTATCGTGACTTGGA \\
\hline PPAR $\alpha$ & ATGGCATCCAGAACAAGGAG & TCCCGTCTTTGTTCATCACA \\
\hline SREBP1 & TGCATTTTCTGACACGCTTC & CCAAGCTGTACAGGCTCTCC \\
\hline TNF- $\alpha$ & TCAGCCTCTTCTCCTTCCTG & TGAGGTACAGGCCCTCTGAT \\
\hline XBP1 & GAAGCCAAGGGGAATGAAGT & GCCCAACAGGATATCAGACTC \\
\hline$\beta$-actin & GCTACAGCTTCACCACCACA & AAGGAAGGCTGGAAAAGAGC \\
\hline
\end{tabular}

ATF4: Activating transcription factor 4; ATF6: activating transcription factor 6; FASn: fatty acid synthase; MTTP: microsomal triglyceride transfer protein; PPAR $\alpha$ : peroxisome proliferator-activated receptor alpha; SREBP1: sterol regulatory element binding protein 1; TNF- $\alpha$ : tumor necrosis factor alpha; XBP1: X-box binding protein 1.

Table 2. List of antibodies and relative dilutions used in Western blotting experiments

\begin{tabular}{ll}
\hline Antibody & Catalog number \\
\hline APOB (1:1000 WB) & ThermoFisher \#HYB0690202 \\
Claudin 1 (1:1000 WB) & ThermoFisher \#2H10D10 37-4900 \\
Claudin 2 (1:1000 WB) & ThermoFisher \#12H12 32-5600 \\
p (Ser800)-TLR4 & ThermoFisher \#PA5-105713 \\
TLR4 & ThermoFisher \#48-2300 \\
ZO-1 (1:500 WB) & ThermoFisher \#33-9100 \\
Vinculin (1:5000 WB) & Abcam \# ab129002 \\
$\beta$-actin (1:5000 WB) & Abcam \#ab6276 \\
\hline
\end{tabular}

\section{Oil red $O$ staining}

Oil red $\mathrm{O}(\mathrm{ORO})$ is a soluble red powder with high affinity for neutral triglycerides and lipids stored in the lipid droplets. In brief, co-cultures were gently washed twice with $1 \mathrm{~mL}$ of sterile PBS $1 \times$, and then cells were fixed with $4 \%$ formalin for $15 \mathrm{~min}$ at room temperature. Next, each sample was washed with $1 \mathrm{~mL}$ of sterile water and $60 \%$ isopropyl alcohol was added for $5 \mathrm{~min}$. ORO working solution was prepared by mixing three parts of ORO stock solution ( $3 \mathrm{mg} / \mathrm{mL}$ in isopropyl alcohol $100 \%$ ) and two parts of sterile water, followed by filtration in a filter funnel. Next, $1 \mathrm{~mL} /$ well of ORO working solution was added to each sample and left for $45 \mathrm{~min}$. Finally, plates were gently rinsed with tap water, paying attention not to disrupt monolayers, and lipid content was visualized in red color. ORO positive areas were quantified by ImageJ software (1.51a version) in 10 random non-overlapping micrographs (at magnification 200x) by calculating the ORO positive area as percentage of pixels above the threshold value with respect to the total pixels per area ${ }^{[32]}$.

\section{Statistical analyses}

Statistical analyses were performed using JMP Pro 16.0 (SAS, Cary, NC) and Prism software (version 9.1.0, GraphPad Software) by using one-way analysis of variance (ANOVA) which was followed by post hoc $t$ tests adjusted for the number of comparisons when multiple groups were involved (Bonferroni correction). The results are shown as means \pm SD or median and interquartile range, for violin plots, of at least three independent experiments, each containing triplicates or quadruplicates. $P$ values lower than 0.05 (at twotailed) were considered statistically significant $\left({ }^{\star *} P<0.0001,{ }^{\star *} P<0.001,{ }^{\star} P<0.01\right)$. 


\section{RESULTS}

\section{Caco-2/HepG2 co-culture system resembles the derangement of intestinal barrier integrity in presence of endotoxin and fatty acids}

To investigate whether Caco-2 polarized monolayers are affected by endotoxin and/or fatty acids, we added in the quiescent medium of the apical chamber $100 \mathrm{ng} / \mathrm{mL}$ LPS and/or a combination of PAOA at the concentration of $0.25 \mathrm{mM}$, to recapitulate the clinical condition of NAFLD patients in which intestinal dysbiosis and excessive FFAs have been reported ${ }^{[10]}$. Then, to test the integrity of Caco-2 confluent monolayers, which recreate intestinal barrier ${ }^{[33]}$, we measured the transepithelial flux of FD4. FD4 permeability across monolayers was increased after the exposure to LPS or PAOA, but more so in the presence of both $(P<0.0001$, one-way ANOVA; adjusted $P<0.0001$ for PAOA LPS $v s$. PAOA or LPS alone; Figure $2 \mathrm{~A}$ ). In keeping with the enhanced permeability, protein expressions of $\mathrm{ZO} 1$, claudin-1, and claudin2, tight-junctions associated proteins, were also decreased $(P<0.0001$, one-way ANOVA; adjusted $P<0.001$ for PAOA LPS $v s$. PAOA or LPS alone; Figure $2 \mathrm{~B}-\mathrm{D}$ ), thus mirroring what occurs in NAFLD patients, in which gut permeability is increased according to the disruption of intercellular tight junctions ${ }^{[12,38]}$.

Caco-2/HepG2 co-cultures secrete TGs in response to FFAs and more so in the co-presence of LPS

We next examined whether Caco-2 monolayers exposed to PAOA and LPS accumulate lipid droplets by exploiting ORO staining to mark neutral lipid content. As expected, Caco-2 stimulated with PAOA displayed enhanced ORO positive areas and intracellular TG content $[P<0.0001$, one-way ANOVA; adjusted $P<0.0001$ for PAOA vs. untreated cells (Ctrl); Figure 3A-C]. However, the co-stimulation of PAOA with LPS did not further exacerbate fat deposition but rather favored TG disposal (adjusted $P<$ 0.0001 for PAOA LPS vs. PAOA alone; Figure 3A-C). Consistently, LPS alone or combined with PAOA enforced TG dismissal, promoting ApoB [Figure 3D], TG-lipoprotein [Figure 3E], TG [Figure 3F], and FFA [Figure $3 \mathrm{G}$ ] release in basolateral media $(P=0.002, P<0.0001, P=0.004$, and $P<0.0001$, one-way ANOVA, respectively; adjusted $P<0.05$ for PAOA LPS $v s$. PAOA for all comparisons). These observations collectively suppor the notion that endotoxemia may boost the secretion of triglyceride-rich lipoproteins, fueling the creation of a pro-inflammatory context and resembling a condition named "lipemia of sepsis" ${ }^{\text {" } 39,40]}$.

\section{HepG2 cells co-cultured with Caco-2 exposed to FFAs and LPS accumulated intracellular lipid droplets}

As a consequence of the enhanced lipid secretion, HepG2 co-cultured with Caco-2 exposed to LPS, PAOA, or both accumulated lipid droplets and increased intracellular TG content $(P<0.0001$, one-way ANOVA; adjusted $P<0.01$ for PAOA LPS vs. PAOA alone; Figure 4A-C), as a result of lipid secretion from Caco-2 polarized monolayers. In this model, intracellular fat accumulation was paralleled by an induction of the lipogenic program, as detected by the conspicuous upregulation of sterol regulatory element binding protein 1-c and fatty acid synthase mRNA levels in HepG2 cells exposed to Caco-2 monolayers stimulated with PAOA and LPS $(P<0.0001$, one-way ANOVA; adjusted $P<0.001$ for PAOA LPS vs. PAOA alone for both comparisons; Figure $4 \mathrm{D}$ and $\mathrm{E}$ ). On the other hand, the expression of genes regulating $\beta$-oxidation, i.e., peroxisome proliferator-activated receptor alpha, and very low-density lipoprotein (VLDL) export, i.e., microsomal triglyceride transfer protein, was suppressed, sustaining fat overload $(P<0.0001$, one-way ANOVA; adjusted $P<0.001$ for PAOA LPS $v s$. PAOA alone for both comparisons; Figure $4 \mathrm{~F}$ and $\mathrm{G}$ ). Superimposable results have previously been reported in patients and in preclinical models of NAFLD ${ }^{[41-43]}$.

\section{Caco-2 polarized monolayers secreted pro-inflammatory cytokines in response to LPS, and the effect was amplified by the co-presence of FFAs}

To ascertain the role of Caco-2 monolayers treated with PAOA and LPS in the promotion of a proinflammatory microenvironment, we quantified cytokine secretion in basolateral media. As expected, TNF- $\alpha$, IL1 $\beta$, and IL6, extensively reported to be induced by endotoxin ${ }^{[44]}$, rose according to the presence of LPS, and even more in the co-presence of FFAs $(P<0.0001$, one-way ANOVA; adjusted $P<0.01$ for PAOA LPS $v s$. PAOA alone for all comparisons; Figure 5A-C). Alongside, LPS per se enforced the phosphorylation 

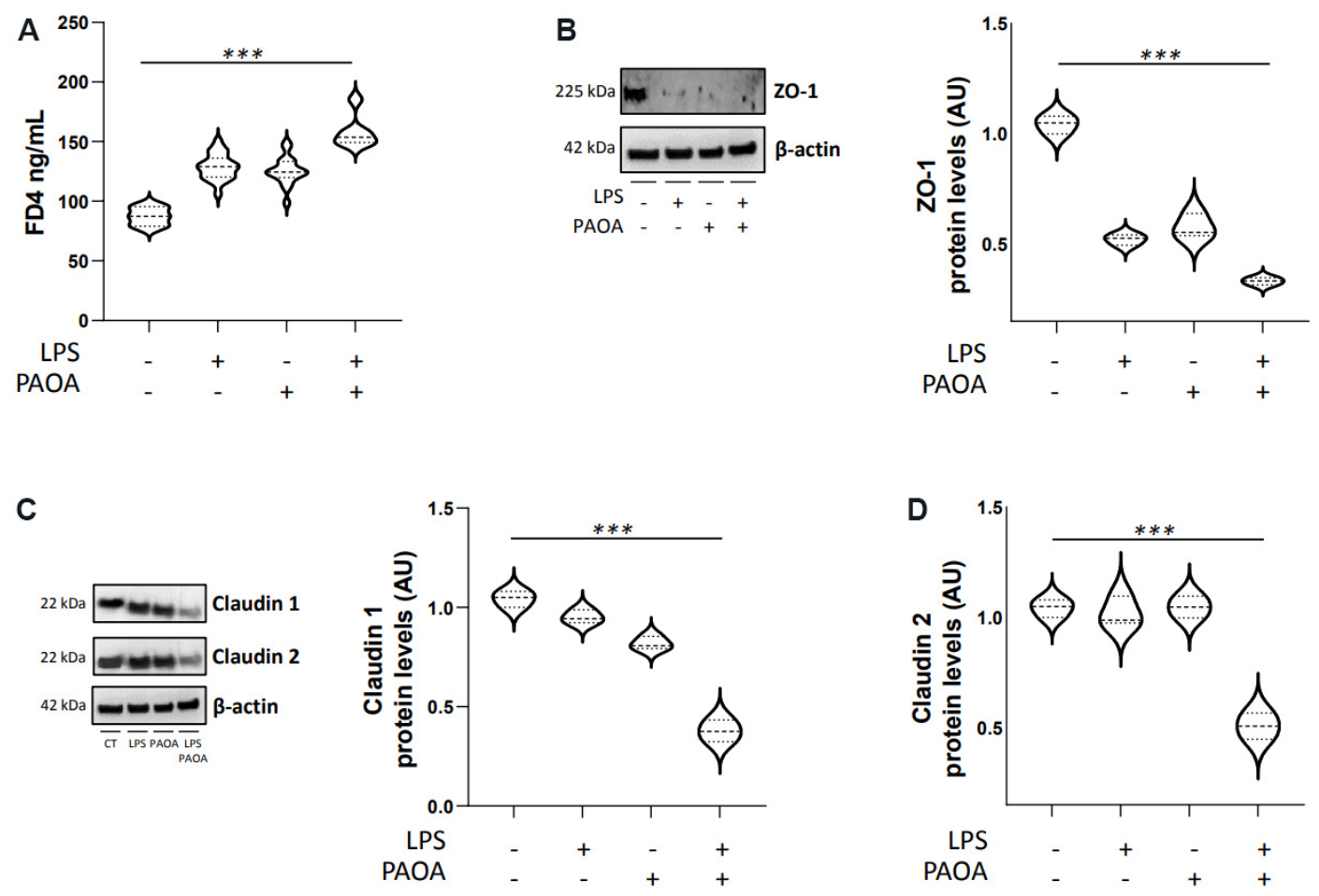

Figure 2. Intestinal permeability was further increased by the co-presence of LPA and PAOA. Polarized monolayers of Caco-2 cells cocultured with HepG2 were assayed for paracellular permeability to $4 \mathrm{kDa}$ FITC-dextrans (FD4). The amount of dextrans diffused from the apical to the basolateral compartments was determined by linear regression at $4 \mathrm{~h}$ using known standard concentrations of FD4. The results are represented as mean \pm standard deviation of three independent experiments in triplicate (A). Tight junction-associated protein levels were evaluated in lysates from Caco-2 cells co-cultured with HepG2 in the presence or not of LPS and/or PAOA using Western blot: ZO-1 (B); Claudin 1 (C); and Claudin 2 (D). The expressions were normalized for $\beta$-actin as the housekeeping gene. For each condition, fresh protein lysates of at least three independent experiments were pooled. ${ }^{\star \star \star} P<0.0001$ according to one-way ANOVA. LPA: Iysophosphatidic acid; PAOA: palmitic and oleic acid; ANOVA: one-way analysis of variance; ZO-1: zonula occludens 1.

of TLR4, stimulating TNF- $\alpha$ gene expression in HepG2 cells $(P<0.001$, one-way ANOVA; adjusted $P<0.01$ for PAOA LPS vs. PAOA alone for both comparisons; Figure 5D and E). Hence, to decipher whether cytokine over-secretion was more attributable to HepG2 cells or to Caco-2 monolayers, we compared mRNA levels of TNF- $\alpha$, a key mediator in the switching from simple steatosis to NASH, between these two cell types and determined that Caco-2 on top more promptly responded to PAOA/LPS combination compared to HepG2 on bottom, further enhancing TNF- $\alpha$ gene expression (adjusted $P<0.0001$ for PAOA LPS condition in Caco-2 monolayer vs. PAOA LPS condition in HepG2 cells; Figure 5E). These findings outline the essential role of the gut-liver axis not only in the development of fatty liver but also in fostering inflammation and consequently the transition towards NASH.

\section{Caco-2/HepG2 co-cultures remodeled the switching from simple steatosis to NASH}

We next assessed the impact of TG accumulation and inflammation driven by intestinal barrier derangement on the development of endoplasmic (ER) and oxidative stress, which are the hallmarks of $\mathrm{NASH}^{[3,45]}$. We demonstrated that HepG2 co-cultured with Caco-2 exposed to a combination of PAOA and LPS strongly upregulated the expression of markers of ER stress and unfolded protein response, including activating transcription factor 4 (ATF4), ATF6, X-box binding protein 1, and GRP78 ( $P=0.0006$ for ATF4 and $P<0.0001$, one-way ANOVA for all genes; adjusted $P<0.05$ for PAOA LPS $v s$. PAOA alone for all comparisons; Figure 6A-D). Oxidative stress was also triggered in HepG2 co-cultured with Caco-2 exposed to a combination of PAOA and LPS, as testified by the elevation of ROS/reactive nitrite species (RNS) 
A

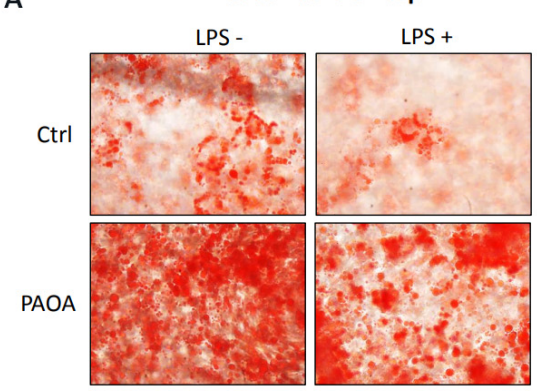

B

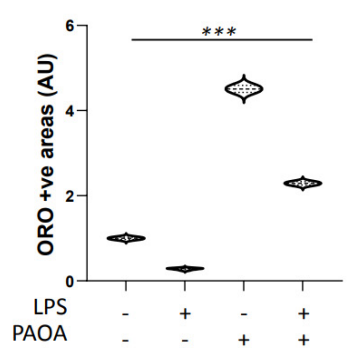

C

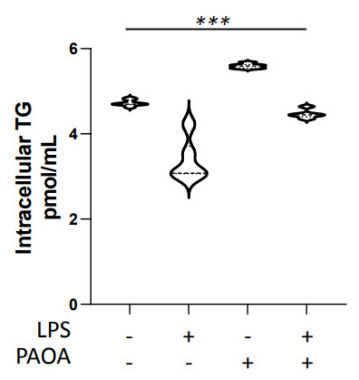

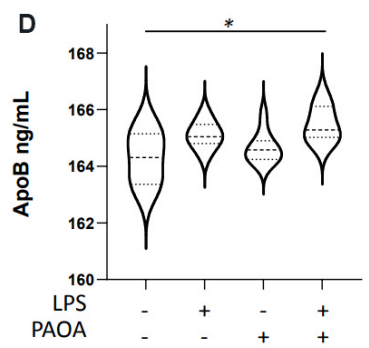
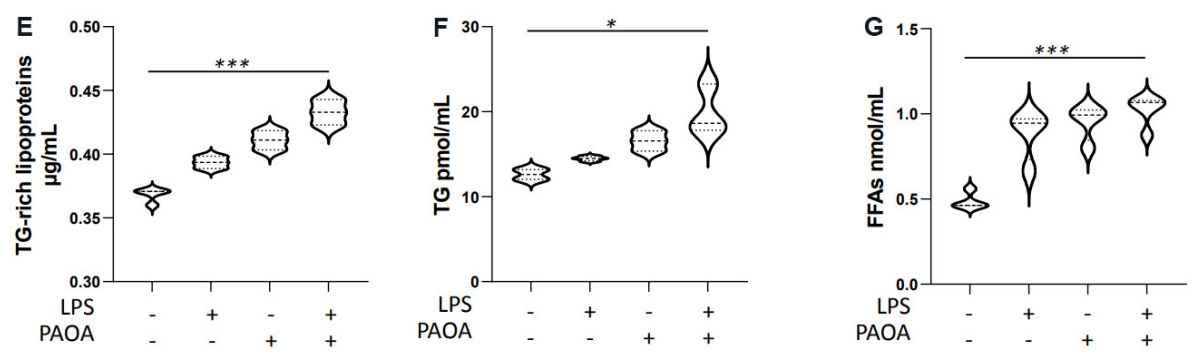

Figure 3. Caco-2 polarized monolayers released TGs in response to FFAs and more so in the co-presence of LPS. Fat accumulation in Caco-2 polarized monolayers on top of the co-culture system was evaluated by ORO staining (200x magnification) and quantified by Image J software in 10 random non-overlapping micrographs for each experimental condition, by calculating the percentage of pixels above the threshold value with respect to the total pixels per area. At least three independent experiments were conducted ( $A, B)$. Intracellular TG content was assessed in lysates from Caco-2 polarized monolayers (C). Assessment of APOB (D), TG-rich lipoproteins (E), TGs (F) and FFAs (G) secreted were measured in concentrated culture media from the basolateral compartment and normalized for the total protein content. For violin plots, data were expressed as median concentration (thick dashed lines) and interquartile range (dotted lines). At least three independent experiments were conducted and pooled together. ${ }^{\star \star \star} P<0.0001,{ }^{\star} P<0.01$ according to oneway ANOVA. TGs: Triglycerides; FFAs: free fatty acids; LPS: lipopolysaccharides; ORO: oil red O; APOB: apolipoprotein B; ANOVA: oneway analysis of variance.

production, $\mathrm{H}_{2} \mathrm{O}_{2}$, aldehyde derivate [malondialdehyde (MDA)] concentrations, and apurinic/apyrimidinic sites, the main ROS-induced DNA damage $(P<0.0001$, one-way ANOVA; adjusted $P<0.01$ for PAOA LPS vs. PAOA alone for all comparisons; Figure $6 \mathrm{E}-\mathrm{H})$. Similarly, the expression of the superoxide dismutase (MnSOD2) was further increased in HepG2 exposed to Caco-2 monolayers treated with PAOA and LPS, possibly as a compensatory mechanism to dampen ROS overflowing $(P<0.0001$, one-way ANOVA; adjusted $P<0.01$ for PAOA LPS vs. PAOA; Figure 6I).

Superimposable findings have been obtained in basolateral media, in which harmful reactive species are elevated as a result of the exposure to PAOA and/or LPS $(P<0.0001$, one-way ANOVA; adjusted $P<0.01$ for PAOA LPS vs. PAOA alone for all comparisons; Supplementary Figure 1A-C). We cannot rule out that these triggers released from both damaged cells may exert a direct detrimental effect on gut barrier, further precipitating the derangement of intestinal tight-junctions integrity.

\section{DISCUSSION}

In this study, we examined the impact of endotoxins and FFAs on the alteration of the intestinal barrier integrity and fat accumulation in hepatocytes, which frequently co-occur in severe NAFLD, by using an in vitro model of Caco-2/HepG2 co-culture, in an attempt to mirror the gut-liver axis. 


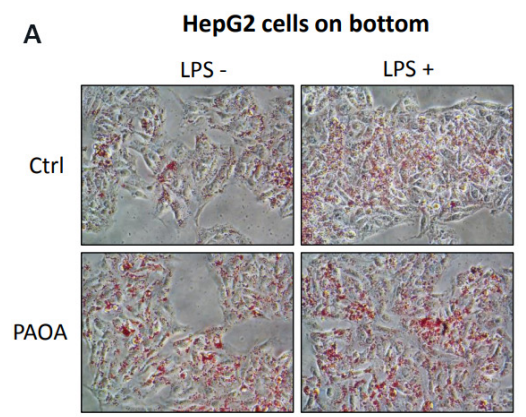

B

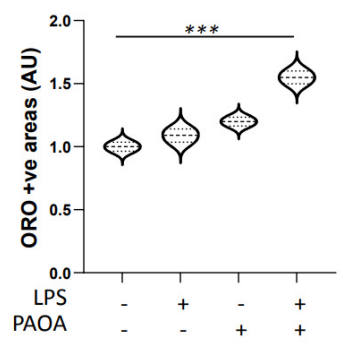

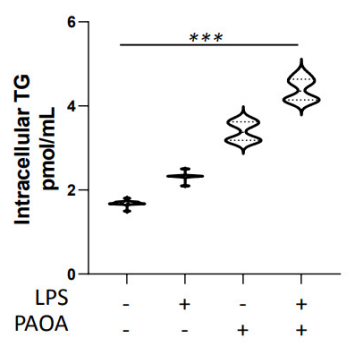
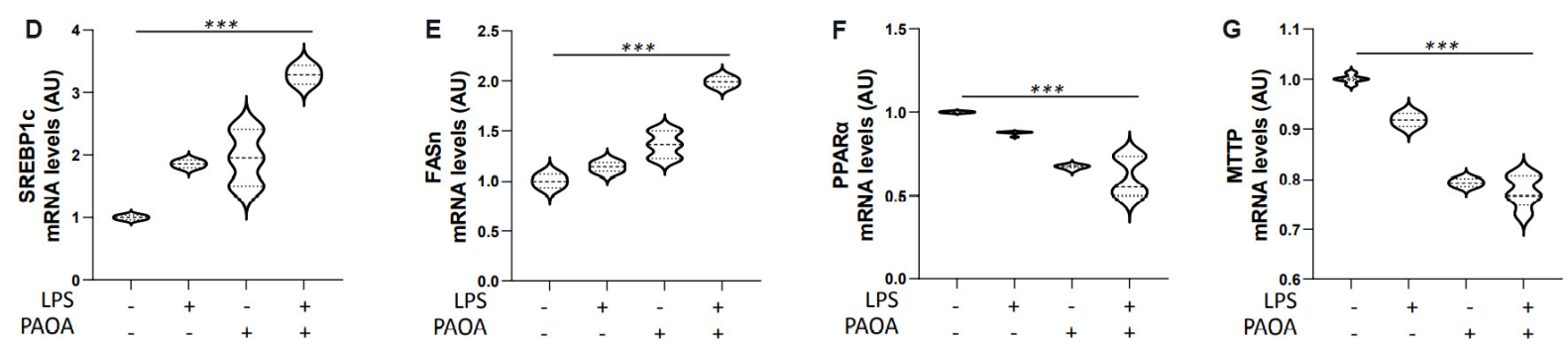

Figure 4. HepG2 cells co-cultured with Caco-2 exposed to FFAs and LPS accumulated intracellular lipid droplets. Fat accumulation in HepG2 on bottom of the co-culture system was evaluated by ORO staining (200x magnification) and quantified by ImageJ software in 10 random non-overlapping micrographs for each experimental condition by calculating the percentage of pixels above the threshold value with respect to the total number of pixels per area. At least three independent experiments were conducted (A, B). Intracellular TG content was assessed in lysates from HepG2 cells (C). (D, E) SREBP1c and FASn (genes involved in de novo lipogenesis); (F) PPAR $\alpha$ (marker of $\beta$-oxidation); (G) and MTTP expressions were evaluated by qRT-PCR in HepG2 cell lysates; mRNA levels were normalized to $\beta$-actin. Data are expressed as fold increase compared to Ctrl. For violin plots, data are expressed as median concentration (thick dashed lines) and interquartile range (dotted lines). At least three independent experiments were conducted and pooled together. ${ }^{\star \star \star} P$ $<0.0001$ according to one-way ANOVA. FFAs: Free fatty acids; LPS: lipopolysaccharides; ORO: oil red O; SREBP1c: sterol regulatory element binding protein 1-c; FASn: fatty acid synthase; PPARa: peroxisome proliferator-activated receptor alpha; MTTP: microsomal triglyceride transfer protein; ANOVA: one-way analysis of variance.

The human intestinal Caco-2 cell line was isolated, for the first time, by colon adenocarcinoma by Fogh et al. ${ }^{[46]}$, and it remains the most powerful tool to reproduce absorptive small intestinal enterocytes in toxicological and pharmacological studies. Indeed, when they are seeded on Transwell filters, Caco-2 confluent monolayers differentiate into their small intestinal phenotype after three weeks of culture, form tight intercellular junctions, and they are fully polarized, acquiring microvilli and the property of lipoprotein secretion ${ }^{[47]}$. In detail, the upper brush border membrane extends microvilli into the apical chamber, as occurs in the intestinal lumen, while the basolateral domain, in contact with the semipermeable porous membrane, is exposed to the lower compartment ${ }^{[30]}$.

HepG2 cells, derived from human hepatocellular carcinoma, are usually used in metabolic studies, since they retain cell differentiation that is lost by primary hepatocytes in culture, preserving their ability to secrete plasma lipoproteins, internalize chylomicrons, regulate cholesterol metabolism and bile acid synthesis, and express inducible cytochrome $\mathrm{P} 450$ pathway ${ }^{[48]}$.

Here, we combined the use of these two different cell types to reproduce as far as possible the gut-liver crosstalk, which occurs in vivo. Our results demonstrate that differentiated Caco-2 monolayers are susceptible to the apical exposure to noxious concentrations of a combination of PAOA and LPS, significantly altering FD4 permeability and tight-junction integrity. Our observations are aligned with the current concept that Western diet and high-fat diet administration in rodents disrupted gut barrier integrity and induced pro-inflammatory luminal LPS transition into the blood flow, contributing to liver 

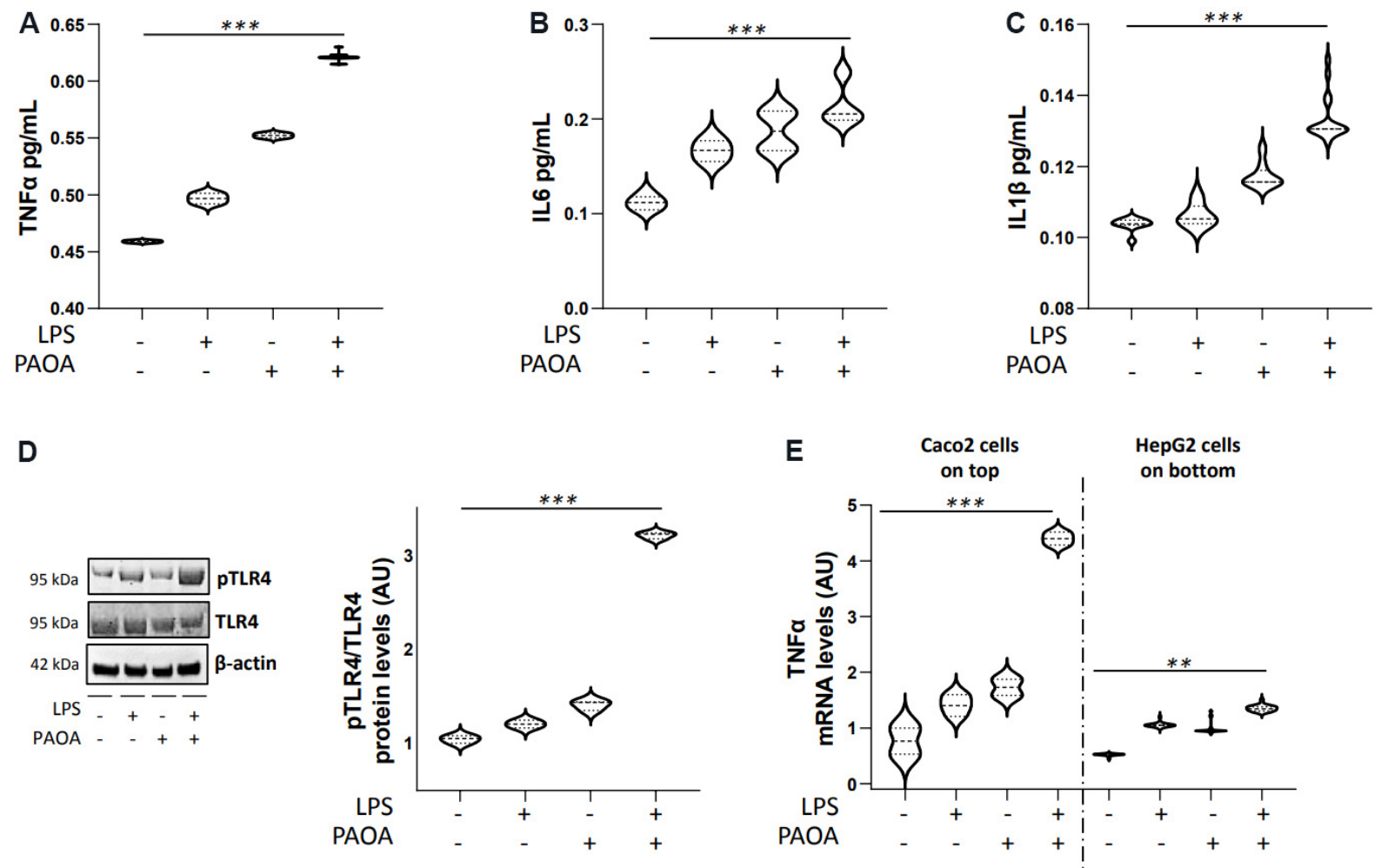

Figure 5. Caco-2 polarized monolayers released pro-inflammatory cytokines in response to LPS and more so in the co-presence of FFAs. Secretion of TNF- $\alpha(A), I L 6(B)$, and IL $1 \beta$ (C) was measured in concentrated culture media from the basolateral compartment and normalized for the total protein content. pTLR4 and total TLR4 protein levels were evaluated in lysates from HepG2 cells co-cultured with Caco-2 stimulated with LPS and/or PAOA using Western blot. The expressions were normalized for the housekeeping gene $\beta$ actin. For each condition, fresh protein lysates of at least three independent experiments were pooled (D). TNF- $\alpha$ mRNA levels were evaluated by qRT-PCR in HepG2 cell lysates and normalized to $\beta$-actin (E). For violin plots, data are expressed as median concentration (thick dashed lines) and interquartile range (dotted lines). At least three independent experiments were conducted and pooled together. ${ }^{\star \star \star} P<0.0001,{ }^{\star \star} P<0.001$ according to one-way ANOVA. LPS: Lipopolysaccharides; FFAs: free fatty acids; TNF- $\alpha$ : tumor necrosis factor alpha; IL6: interleukin 6; TLR4: toll-like receptor 4; PAOA: palmitic and oleic acid; ANOVA: one-way analysis of variance.

damage ${ }^{[13,14,49]}$. Moreover, the incubation of HepG2 cultures with effectors released in basolateral media from Caco-2 boosts fat deposition, paralleled by TLR4 activation and severe oxidative damage, resulting in lipid peroxidation and ROS-triggered DNA injuries. Our findings confirm that elevated concentration of serum LPS forces the activation of TLR4/NF- $\mathrm{kB}$ signaling in hepatocytes, thus driving, in turn, pro-inflammatory cytokine secretion and ROS production.

The relevance of gut-liver interplay in NAFLD susceptibility has been broadly addressed in both preclinical and clinical studies, although it remains a matter of debate. Despite the relatively standard co-culture conditions that we chose for the growth and maintenance of the Caco-2 and HepG2 cell lines, this is the first study, to our knowledge, that adopts this system to resemble human NAFLD and deeply investigate the mechanisms underlying NASH development. Our co-culture method is surely technically limited compared to novel and more advanced three-dimensional cell culture protocols (i.e., spheroids, organoids, 3D bioprinted tissues, and many others), however it seems to be fully reproducible, cost-effective, and capable of mirroring the interactions between the intestinal tract and the liver during the natural history of human disease. 

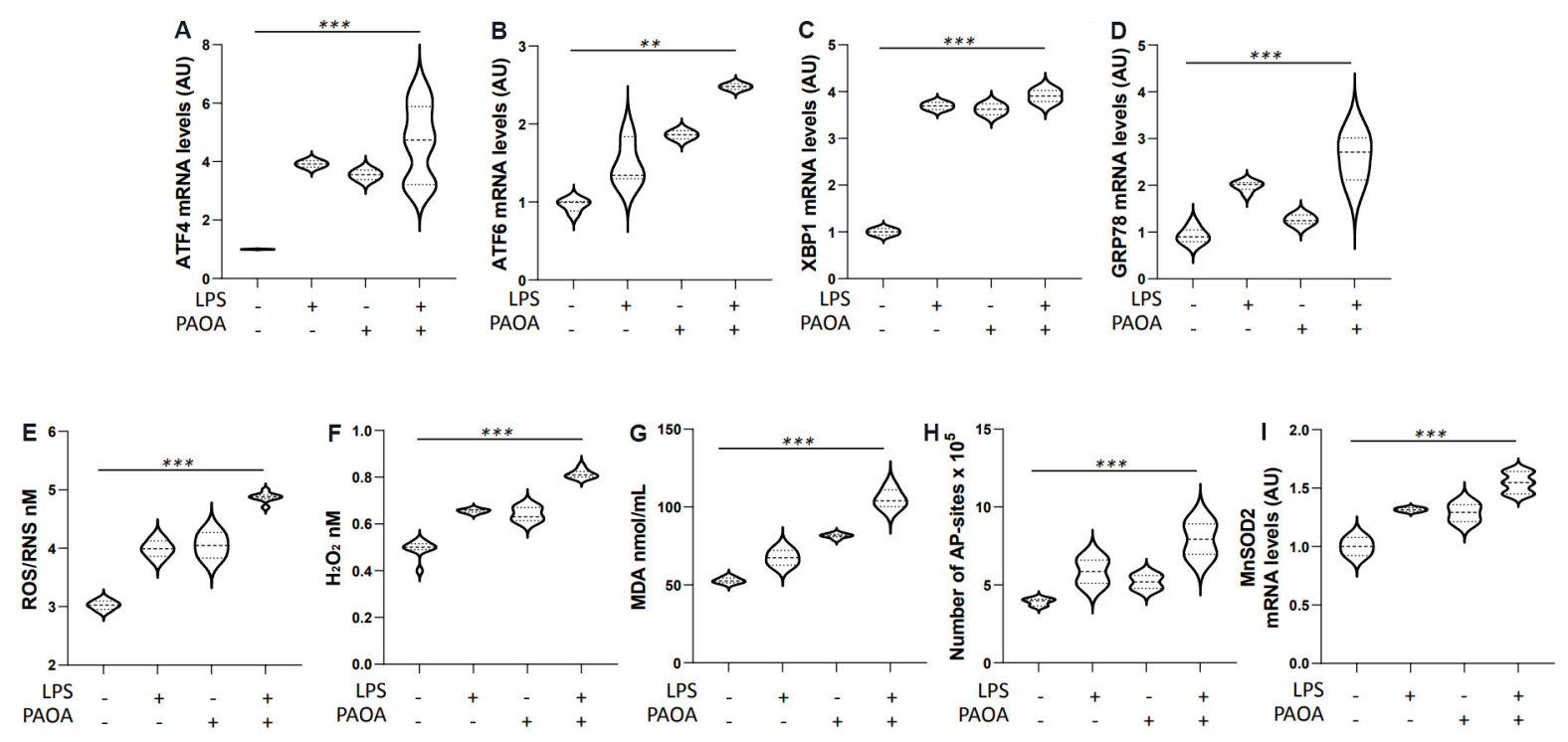

Figure 6. HepG2 cells co-cultured with Caco-2 exposed to FFAs and LPS displayed an enhanced ER and oxidative stress. The expressions of ATF4 (A), ATF6 (B), XBP1 (C), and GRP78 (D), markers of ER stress were evaluated by qRT-PCR in HepG2 cell lysates and normalized using $\beta$-actin as the housekeeping gene. Data are expressed as fold increase compared to Ctrl. ROS/RNS content was measured using a colorimetric assay (Abcam) (E). $\mathrm{H}_{2} \mathrm{O}_{2}$ levels were quantified in $\mathrm{HepG} 2$ lysates through DCF ROS/RNS Colorimetric Assay Kit (Abcam) (F). Malondialdehyde (MDA) was colorimetrically measured in HepG2 lysates following the manufacturer's instructions (Abcam) $(G)$. The number of apurinic/apyrimidinic (AP) sites was obtained by isolating total DNA from HepG2 lysates and then it was normalized to the total amount of DNA extracted $(H)$. MnSOD2 mRNA levels were assessed by qRT-PCR and normalized to $\beta$-actin (I). For violin plots, data are expressed as median concentration (thick dashed lines) and interquartile range (dotted lines). At least three independent experiments were conducted and pooled together. ${ }^{\star \star \star} P<0.0001,{ }^{\star \star} P<0.001$ according to one-way ANOVA. FFAs: Free fatty acids; LPS: lipopolysaccharides; ER: endoplasmic reticulum; ATF4: activating transcription factor 4; ATF6: activating transcription factor 6; XBP1: X-box binding protein 1; ROS: reactive oxygen species; RNS: reactive nitrite species; ANOVA: one-way analysis of variance.

\section{CONCLUSION}

The Caco-2/HepG2 co-culture system may faithfully reproduce the breach in the intestinal barrier integrity that occurs in NAFLD, thus resulting in the increased inflammatory response and oxidative and ER stress, which in turn promote the switch towards NASH. This study paves the way for future investigations, exploiting co-culture platforms to pinpoint precise cell-to-cell communications and soluble mediators exchanged between different cell types during progressive NAFLD onset and to test the efficacy of novel therapeutic strategies or dietary approaches to this disease.

\section{DECLARATIONS}

\section{Authors' contributions}

Writing the manuscript, preparing figures, read and approved the final draft: Meroni M, Paolini E, Longo M, Piciotti R, Tria G, Fargion S, Fracanzani AL, Dongiovanni P

All authors have read and agreed with the published version of the manuscript.

\section{Availability of data and materials}

Not applicable.

\section{Financial support and sponsorship}

The study was supported by Ricerca Corrente Fondazione IRCCS Cà Granda and Ricerca Finalizzata Ministero della Salute GR-2019-12370172. 


\section{Conflicts of interest}

All authors declared there are no conflicts of interest.

\section{Ethical approval and consent to participate}

Not applicable.

\section{Consent for publication}

Not applicable.

\section{Copyright}

(c) The Author(s) 2022.

\section{REFERENCES}

1. Meroni M, Longo M, Paolini E, et al. MAFLD definition underestimates the risk to develop HCC in genetically predisposed patients. $J$ Intern Med ;2021:374-6. DOI PubMed

2. Younossi Z, Henry L. Contribution of alcoholic and nonalcoholic fatty liver disease to the burden of liver-related morbidity and mortality. Gastroenterology 2016;150:1778-85. DOI PubMed

3. Eslam M, Sanyal AJ, George J; International Consensus Panel. MAFLD: a consensus-driven proposed nomenclature for metabolic associated fatty liver disease. Gastroenterology 2020;158:1999-2014.e1. DOI PubMed

4. Younossi ZM, Koenig AB, Abdelatif D, Fazel Y, Henry L, Wymer M. Global epidemiology of nonalcoholic fatty liver disease-Metaanalytic assessment of prevalence, incidence, and outcomes. Hepatology 2016;64:73-84. DOI PubMed

5. Wong RJ, Aguilar M, Cheung R, et al. Nonalcoholic steatohepatitis is the second leading etiology of liver disease among adults awaiting liver transplantation in the United States. Gastroenterology 2015;148:547-55. DOI PubMed

6. Dongiovanni P, Meroni M, Longo M, Fargion S, Fracanzani AL. Genetics, immunity and nutrition boost the switching from NASH to HCC. Biomedicines 2021;9:1524. DOI PubMed PMC

7. Tilg H, Moschen AR. Evolution of inflammation in nonalcoholic fatty liver disease: the multiple parallel hits hypothesis. Hepatology 2010;52:1836-46. DOI PubMed

8. Meroni M, Longo M, Dongiovanni P. Alcohol or gut microbiota: who is the guilty? Int J Mol Sci 2019;20:4568. DOI PubMed PMC

9. Poeta M, Pierri L, Vajro P. Gut-liver axis derangement in non-alcoholic fatty liver disease. Children (Basel) 2017;4:66. DOI PubMed PMC

10. Meroni M, Longo M, Dongiovanni P. The role of probiotics in nonalcoholic fatty liver disease: a new insight into therapeutic strategies. Nutrients 2019;11:2642. DOI PubMed PMC

11. Giorgio V, Miele L, Principessa L, et al. Intestinal permeability is increased in children with non-alcoholic fatty liver disease, and correlates with liver disease severity. Dig Liver Dis 2014;46:556-60. DOI PubMed

12. Miele L, Valenza V, La Torre G, et al. Increased intestinal permeability and tight junction alterations in nonalcoholic fatty liver disease. Hepatology 2009;49:1877-87. DOI PubMed

13. Lindstedt G, Lindstedt S, Gustafsson BE. Mucus in intestinal contents of germfree rats. J Exp Med 1965;121:201-13. DOI PubMed PMC

14. Wang L, Fouts DE, Stärkel P, et al. Intestinal REG3 lectins protect against alcoholic steatohepatitis by reducing mucosa-associated microbiota and preventing bacterial translocation. Cell Host Microbe 2016;19:227-39. DOI PubMed PMC

15. Cani PD, Osto M, Geurts L, Everard A. Involvement of gut microbiota in the development of low-grade inflammation and type 2 diabetes associated with obesity. Gut Microbes 2012;3:279-88. DOI PubMed PMC

16. Boulangé CL, Neves AL, Chilloux J, Nicholson JK, Dumas ME. Impact of the gut microbiota on inflammation, obesity, and metabolic disease. Genome Med 2016;8:42. DOI PubMed PMC

17. Del Chierico F, Nobili V, Vernocchi P, et al. Gut microbiota profiling of pediatric nonalcoholic fatty liver disease and obese patients unveiled by an integrated meta-omics-based approach. Hepatology 2017;65:451-64. DOI PubMed

18. Delarue J, Lallès JP. Nonalcoholic fatty liver disease: roles of the gut and the liver and metabolic modulation by some dietary factors and especially long-chain n-3 PUFA. Mol Nutr Food Res 2016;60:147-59. DOI PubMed

19. Bibbò S, Ianiro G, Dore MP, Simonelli C, Newton EE, Cammarota G. Gut microbiota as a driver of inflammation in nonalcoholic fatty liver disease. Mediators Inflamm 2018;2018:9321643. DOI PubMed PMC

20. Puri P, Sanyal AJ. The intestinal microbiome in nonalcoholic fatty liver disease. Clin Liver Dis 2018;22:121-32. DOI PubMed

21. Borrelli A, Bonelli P, Tuccillo FM, et al. Role of gut microbiota and oxidative stress in the progression of non-alcoholic fatty liver disease to hepatocarcinoma: current and innovative therapeutic approaches. Redox Biol 2018;15:467-79. DOI PubMed PMC

22. Buzzetti E, Pinzani M, Tsochatzis EA. The multiple-hit pathogenesis of non-alcoholic fatty liver disease (NAFLD). Metabolism 2016;65:1038-48. DOI PubMed

23. Schnabl B, Brenner DA. Interactions between the intestinal microbiome and liver diseases. Gastroenterology 2014;146:1513-24. DOI PubMed PMC

24. Ferreira DF, Fiamoncini J, Prist IH, Ariga SK, de Souza HP, de Lima TM. Novel role of TLR4 in NAFLD development: modulation of 
metabolic enzymes expression. Biochim Biophys Acta 2015;1851:1353-9. DOI PubMed

25. Cai C, Zhu X, Li P, et al. NLRP3 deletion inhibits the non-alcoholic steatohepatitis development and inflammation in Kupffer cells induced by palmitic acid. Inflammation 2017;40:1875-83. DOI PubMed

26. Ray K. NAFLD. Leaky guts: intestinal permeability and NASH. Nat Rev Gastroenterol Hepatol 2015;12:123. DOI PubMed

27. Meroni M, Longo M, Rametta R, Dongiovanni P. Genetic and epigenetic modifiers of alcoholic liver disease. Int J Mol Sci 2018;19:3857. DOI PubMed PMC

28. De Gregorio V, Telesco M, Corrado B, et al. Intestine-liver axis on-chip reveals the intestinal protective role on hepatic damage by emulating ethanol first-pass metabolism. Front Bioeng Biotechnol 2020;8:163. DOI PubMed PMC

29. Breemen RB, Li Y. Caco-2 cell permeability assays to measure drug absorption. Expert Opin Drug Metab Toxicol 2005;1:175-85. DOI PubMed

30. Garcia MN, Flowers C, Cook JD. The Caco-2 cell culture system can be used as a model to study food iron availability. J Nutr 1996;126:251-8. DOI PubMed

31. Tayyeb JZ, Popeijus HE, Mensink RP, Plat J. Butyric acid added apically to intestinal Caco-2 cells elevates hepatic ApoA-I transcription and rescues lower ApoA-I expression in inflamed HepG2 cells co-cultured in the basolateral compartment. Biomolecules 2021;11:71. DOI PubMed PMC

32. Meroni M, Dongiovanni P, Longo M, et al. Mboat7 down-regulation by hyper-insulinemia induces fat accumulation in hepatocytes. EBioMedicine 2020;52:102658. DOI PubMed PMC

33. Sambuy Y, De Angelis I, Ranaldi G, Scarino ML, Stammati A, Zucco F. The Caco-2 cell line as a model of the intestinal barrier: influence of cell and culture-related factors on Caco-2 cell functional characteristics. Cell Biol Toxicol 2005;21:1-26. DOI PubMed

34. Twarog C, Liu K, O'Brien PJ, et al. A head-to-head Caco-2 assay comparison of the mechanisms of action of the intestinal permeation enhancers: SNAC and sodium caprate $\left(\mathrm{C}_{10}\right)$. Eur J Pharm Biopharm 2020;152:95-107. DOI PubMed

35. He S, Liu F, Xu L, et al. Protective effects of ferulic acid against heat stress-induced intestinal epithelial barrier dysfunction in vitro and in vivo. PLoS One 2016;11:e0145236. DOI PubMed PMC

36. Longo M, Meroni M, Paolini E, et al. TM6SF2/PNPLA3/MBOAT7 loss-of-function genetic variants impact on NAFLD development and progression both in patients and in in vitro models. Cell Mol Gastroenterol Hepatol 2021;13:759-88. DOI PubMed PMC

37. Longo M, Paolini E, Meroni M, et al. $\alpha$-lipoic acid improves hepatic metabolic dysfunctions in acute intermittent porphyria: a proofof-concept study. Diagnostics (Basel) 2021;11:1628. DOI PubMed PMC

38. Kessoku T, Kobayashi T, Tanaka K, et al. The role of leaky gut in nonalcoholic fatty liver disease: a novel therapeutic target. Int J Mol Sci 2021;22:8161. DOI PubMed PMC

39. Barcia AM, Harris HW. Triglyceride-rich lipoproteins as agents of innate immunity. Clin Infect Dis 2005;41 Suppl 7:S498-503. DOI PubMed

40. Harris HW, Gosnell JE, Kumwenda ZL. The lipemia of sepsis: triglyceride-rich lipoproteins as agents of innate immunity. $J$ Endotoxin Res 2000;6:421-30. PubMed

41. Meroni M, Longo M, Erconi V, et al. mir-101-3p downregulation promotes fibrogenesis by facilitating hepatic stellate cell transdifferentiation during insulin resistance. Nutrients 2019;11:2597. DOI PubMed PMC

42. Dongiovanni P, Meroni M, Baselli GA, et al. Insulin resistance promotes Lysyl Oxidase Like 2 induction and fibrosis accumulation in non-alcoholic fatty liver disease. Clin Sci (Lond) 2017;131:1301-15. DOI PubMed

43. Parlati L, Régnier M, Guillou H, Postic C. New targets for NAFLD. JHEP Rep 2021;3:100346. DOI PubMed PMC

44. Ceccarelli S, Panera N, Mina M, et al. LPS-induced TNF- $\alpha$ factor mediates pro-inflammatory and pro-fibrogenic pattern in nonalcoholic fatty liver disease. Oncotarget 2015;6:41434-52. DOI PubMed PMC

45. Ashraf NU, Sheikh TA. Endoplasmic reticulum stress and Oxidative stress in the pathogenesis of Non-alcoholic fatty liver disease. Free Radic Res 2015;49:1405-18. DOI PubMed

46. Fogh J, Fogh JM, Orfeo T. One hundred and twenty-seven cultured human tumor cell lines producing tumors in nude mice. $J$ Natl Cancer Inst 1977;59:221-6. DOI PubMed

47. Ferruzza S, Rossi C, Scarino ML, Sambuy Y. A protocol for differentiation of human intestinal Caco-2 cells in asymmetric serumcontaining medium. Toxicol In Vitro 2012;26:1252-5. DOI PubMed

48. Javitt NB. Hep G2 cells as a resource for metabolic studies: lipoprotein, cholesterol, and bile acids. FASEB J 1990;4:161-8. DOI PubMed

49. Brahe LK, Astrup A, Larsen LH. Is butyrate the link between diet, intestinal microbiota and obesity-related metabolic diseases? Obes Rev 2013;14:950-9. DOI PubMed 